\title{
Angioplastia Coronária em Paciente com Infarto do Miocárdio e Púrpura Trombocitopênica Idiopática
}

\author{
Felipe Augusto Ortencio'1, leda Maria Liguori', Mauro Guimarães Albuquerque1, \\ Ana Rita de Araujo Burgos¹, Rosa Maria Santos Tabosa do Egito', Enilton Sergio Tabosa do Egito", \\ Leopoldo Soares Piegas ${ }^{1,2}$, Edson Renato Romano ${ }^{1}$, Adriana Moreira ${ }^{1}$, \\ J. Ribamar Costa Jr. ${ }^{1,2}$, J. Eduardo Sousa ${ }^{1,2}$
}

\section{RESUMO}

A púrpura trombocitopênica idiopática (PTI) é um distúrbio autoimune, caracterizado clinicamente por plaquetopenia e sangramentos mucocutâneos. Trata-se de doença rara na população geral e a ocorrência de infarto agudo do miocárdio (IAM) em pacientes com PTI é ainda menos frequente. Neste artigo os autores descrevem um caso com PTI no qual foi praticada angioplastia coronária transluminal na fase evolutiva do IAM.
DESCRITORES: Púrpura trombocitopênica idiopática. Angioplastia transluminal percutânea coronária. Infarto do miocárdio.
A púrpura trombocitopênica idiopática, também conhecida como púrpura trombocitopênica imune ou doença de Werlhof ${ }^{1}$, é uma doença rara, com incidência na população geral variando de 1,6 caso a 15,5 casos por 100 mil pessoas por ano. Sua etiologia é autoimune e caracterizada pela presença de autoanticorpos que se ligam na superfície das plaquetas, levando a seu sequestro no sistema reticuloendotelial. Manifesta-se clinicamente por baixa contagem de plaquetas e tendência a sangramentos mucocutâneos..$^{2-4}$

\footnotetext{
Hospital do Coração da Associação do Sanatório Sírio - São Paulo, SP, Brasil.

2 Instituto Dante Pazzanese de Cardiologia - São Paulo, SP, Brasil. Correspondência: Ieda Maria Liguori. Rua Desembargador Eliseu Guilherme, 123/147 - Paraíso - São Paulo, SP, Brasil - CEP 04004-030 E-mail: iliguori@hcor.com.br

Recebido em: 30/8/2010 • Aceito em: 27/9/2010
}

\section{ABSTRACT}

\author{
Coronary Angioplasty in a Patient \\ with Myocardial Infarction and \\ Idiopathic Thrombocytopenic Purpura
}

Idiopathic thrombocytopenic purpura (ITP) is an autoimmune disorder characterized by the presence of thrombocytopenia and mucocutaneous bleeding. It is a rare condition and the occurrence of an acute myocardial infarction (AMI) in patients with ITP is even less common. In the present manuscript the authors report a patient with ITP who underwent percutaneous transluminal coronary angioplasty in the follow-up phase of an AMI.

KEY-WORDS: Purpura, thrombocytopenic, idiopathic. Angioplasty, transluminal, percutaneous coronary. Myocardial infarction.
A coexistência de púrpura trombocitopênica idiopática com doença aterosclerótica é possível, porém sua associação com infarto agudo do miocárdio é incomum, em decorrência do papel decisivo que as plaquetas desempenham nos eventos trombóticos. O tratamento nesse cenário é um desafio, uma vez que a antiagregação e a anticoagulação aumentam o risco de sangramento nesses pacientes, e o uso de trombolíticos é contraindicado. ${ }^{5-7}$

\section{RELATO DO CASO}

Paciente com 62 anos de idade, do sexo masculino, com história pregressa de revascularização do miocárdio em 2001, com enxertos da artéria mamária interna esquerda para a artéria descendente anterior e quatro enxertos de veia safena para as artérias diagonal, marginal, ventricular posterior da circunflexa e coronária direita. Apresentava como fatores de risco para aterosclerose hipertensão arterial sistêmica, hipercolesterolemia e diabetes. O diagnóstico de púrpura trombocitopênica 
idiopática foi realizado no pós-operatório tardio e, desde então, o paciente estava em uso da terapia preconizada, corticoterapia (prednisona $5 \mathrm{mg} / \mathrm{dia}$ ), mantendo-se com contagem de plaquetas estável em torno de $100.000 / \mathrm{mm}^{3}$.

O paciente foi admitido no Hospital do Coração da Associação do Sanatório Sírio em maio de 2010, com quadro clínico de infarto agudo do miocárdio com supradesnivelamento do segmento ST, com alterações do eletrocardiograma em derivações da parede inferior e precordiais direitas. A creatinina sérica era normal $(1,1 \mathrm{mg} / \mathrm{dl})$ e o ecocardiograma transtorácico demonstrou hipocinesia grave da parede inferosseptal do ventrículo esquerdo, hipocinesia moderada lateroapical do ventrículo direito e fração de ejeção de 43\%. O pico de creatina quinase fração $M B(C K-M B)$ massa foi de 52,2 ng/ml (normal $<5,1$ ) e de troponina I, de $20 \mathrm{ng} / \mathrm{ml}$ (normal < 0,2).

A cinecoronariografia realizada logo após a admissão evidenciou oclusão das artérias descendente anterior, circunflexa e coronária direita e dos enxertos de veia safena para as artérias marginal e ventricular posterior da circunflexa. Os enxertos da artéria mamária interna esquerda para a artéria descendente anterior e de veia safena para a artéria diagonal estavam pérvios, com bom fluxo. A ponte de safena para a artéria coronária direita, entretanto, exibia obstrução de $80 \%$ na porção proximal do segmento venoso e fluxo TIMI III.

O paciente foi mantido em uso de nitroglicerina por via endovenosa, ácido acetilsalicílico (100 mg/dia), clopidogrel (dose de ataque de 600 mg e manutenção de $75 \mathrm{mg} / \mathrm{dia}$ ), atenolol (50 mg/dia), captopril (75 mg/dia), atorvastatina (40 mg/dia) e insulina regular por via subcutânea. A dose diária de prednisona foi aumentada de $5 \mathrm{mg}$ para $20 \mathrm{mg}$, no segundo dia de internação. Em nenhum momento foi utilizada anticoagulação préprocedimento com heparina.

A contagem de plaquetas manteve-se em torno de $100.000 / \mathrm{mm}^{3}$ e no quarto dia o paciente foi submetido com sucesso a intervenção coronária percutânea, com utilização de filtro de proteção distal e implante de dois stents não-farmacológicos Liberté 4 × 12 mm nos terços proximal e médio na ponte de safena para a coronária direita (Figura 1). Nenhum sangramento ocorreu e o paciente recebeu alta assintomático, três dias após a intervenção coronária percutânea.

\section{DISCUSSÃO}

São poucos os casos publicados de associação de púrpura trombocitopênica idiopática e infarto agudo do miocárdio, e até o momento não existem recomendações para o manuseio desses pacientes, devendo ser realizada cuidadosa escolha de antiplaquetários e anticoagulantes e utilizada a melhor estratégia de revascularização.
A trombólise, um dos pilares do tratamento do infarto agudo do miocárdio com supradesnivelamento do segmento ST, está contraindicada em decorrência da diátese hemorrágica. Já os antiplaquetários podem ser administrados, nas doses de ataque e de manutenção usuais. ${ }^{4-6,8}$ Entre os tienopiridínicos, o clopidogrel é considerado mais seguro que a ticlopidina, pela menor incidência de efeitos hematológicos adversos. É importante monitorar o número de plaquetas nesses pacientes, especialmente nos primeiros três meses, e acompanhar o aparecimento eventual de sangramentos, que pode acarretar a suspensão desses fármacos. ${ }^{5}$

A utilização de heparina não-fracionada ou heparina de baixo peso molecular para pacientes com risco de sangramento é motivo de preocupação, pelo risco do desenvolvimento de trombocitopenia induzida pela heparina. O fondaparinux, um inibidor indireto do fator Xa, ao contrário da heparina não-fracionada e da heparina de baixo peso molecular, não tem efeito nas plaquetas e pode reduzir o risco de sangramentos nesses pacientes. Tem eficácia semelhante à da enoxaparina no que se refere à redução dos eventos isquêmicos na estabilização clínica da síndrome coronária aguda sem supradesnivelamento do segmento ST. Sua utilização isolada, como terapêutica adjunta à intervenção coronária percutânea, no entanto, pode aumentar as chances de aparecimento de trombos no cateter. Assim, heparina não-fracionada deve ser acrescentada ao esquema antitrombótico dos pacientes encaminhados a angiografia e a intervenção coronária percutânea na síndrome coronária aguda sem supradesnivelamento do segmento ST, estando o fondaparinux contraindicado na intervenção coronária percutânea primária. Alternativa ainda não disponível no Brasil é a bivalirudina, um inibidor direto da trombina que pode ser utilizado isoladamente tanto na estabilização clínica de pacientes com síndrome coronária aguda sem supradesnivelamento do segmento ST como durante a intervenção coronária percutânea no infarto agudo do miocárdio com supradesnivelamento do segmento ST. A bivalirudina é superior à associação heparina nãofracionada/inibidor da glicoproteína Ilb/Illa na intervenção coronária percutânea primária, por reduzir sangramentos sem aumentar os eventos isquêmicos. ${ }^{9}$

Em relação à revascularização miocárdica, há relatos na literatura de revascularização cirúrgica bem-sucedida em pacientes com púrpura trombocitopênica idiopática, mas nenhum deles na vigência de infarto agudo do miocárdio com supradesnivelamento do segmento ST. Em situações eletivas, deve ser realizado pré-tratamento com gamaglobulina endovenosa quatro a cinco dias antes do procedimento, para aumentar o número de plaquetas e diminuir as chances de sangramento. ${ }^{3}$ Em nosso caso, o antecedente de revascularização cirúrgica prévia associado à identificação de lesão com morfologia adequada à intervenção coronária percutânea na cinecoronariografia fez com que essa opção terapêutica fosse escolhida. Na Tabela 1 
Ortencio FA, et al. Angioplastia Coronária em Paciente com Infarto do Miocárdio e Púrpura Trombocitopênica Idiopática. Rev Bras Cardiol Invasiva. 2010;18(3):354-7.
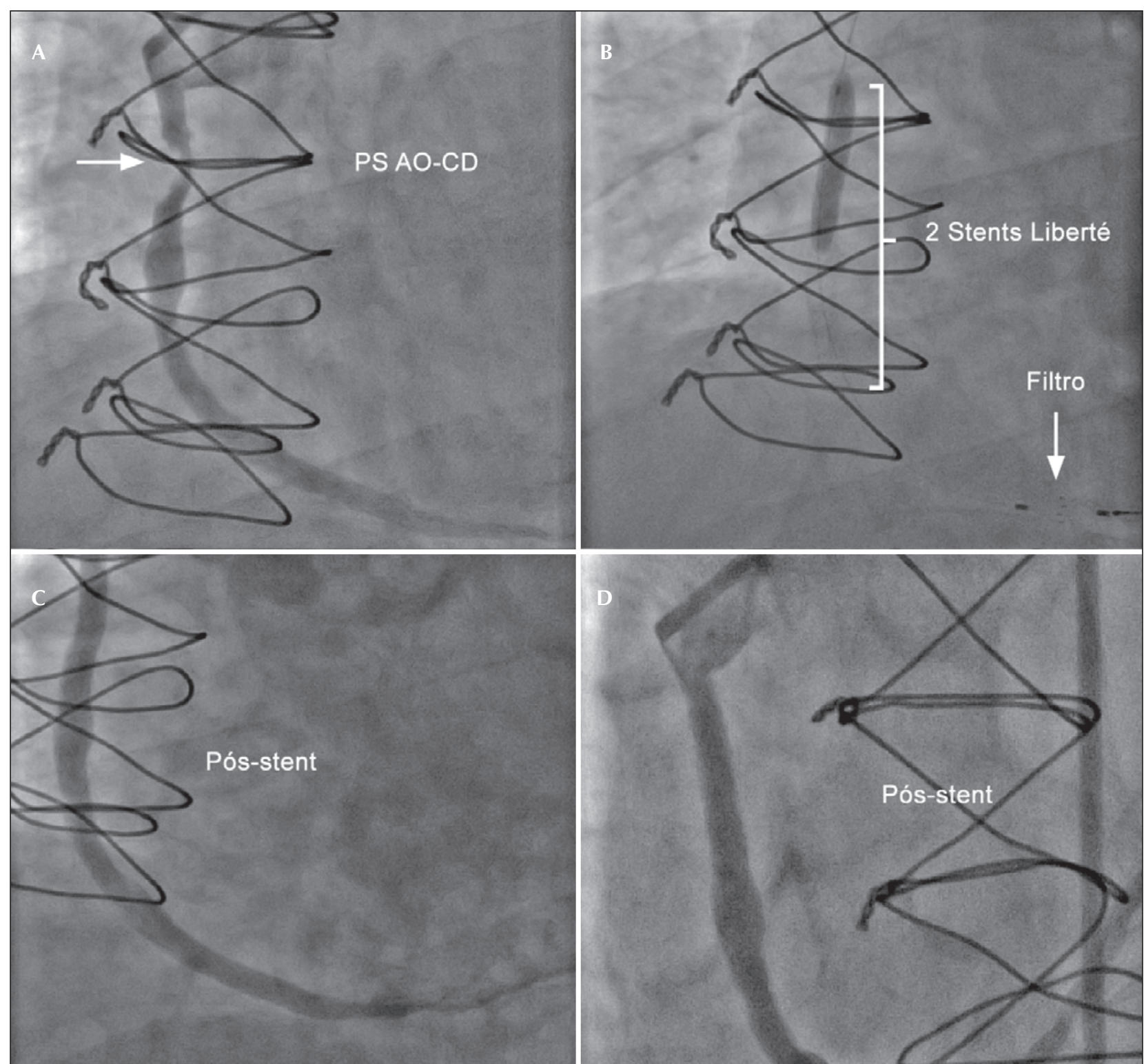

Figura 1 - Paciente com púrpura trombocitopênica idiopática tratado com stent não-farmacológico na fase aguda do infarto do miocárdio. Em A, lesão na ponte de safena para a artéria coronária direita. Em B, implante dos stents com colocação prévia do filtro de proteção. Em C e D, aspecto da ponte de safena após o implante dos stents não-farmacológicos. $\mathrm{PS} A \mathrm{AO} C \mathrm{CD}=$ ponte de safena para a artéria coronária direita.

listamos os poucos relatos de caso de intervenção coronária percutânea no infarto agudo do miocárdio com supradesnivelamento do segmento ST em pacientes com púrpura trombocitopênica idiopática.

A escolha do tipo de stent a ser implantado deve ser objeto de cuidadosa consideração. A utilização de um stent não-farmacológico nos pareceu a melhor opção nesse caso, em decorrência da necessidade de utilizar terapêutica antiplaquetária dupla por apenas um mês. Adicionalmente, no manuseio dos enxertos durante o procedimento, devem ser consideradas também as abordagens que diminuem as chances de embolização distal de debris, como os filtros de proteção distal e os stents dedicados, revestidos por malhas poliméricas, classes de recomendação I e Ilb no Guidelines on Myocardial Revascularization do ESC/EACTS Guidelines, respectivamente. ${ }^{11}$

Por fim, a utilização do acesso radial tem mostrado reduzir a incidência de sangramentos. Meta-análise recente demonstrou que a via radial reduz sangramentos maiores, quando comparada à via femoral, em $73 \%[0,05 \%$ vs. $2,3 \%$, odds ratio (OR) 0,27 , intervalo de confiança de 95\% (IC 95\%) 0,16-0,45; P < 0,001], apesar de maior chance de insucesso do procedimento por não se conseguir ultrapassar a lesão por meio de guia, balão ou stent durante a intervenção coronária 
TABELA 1

Angioplastia coronária na fase aguda do infarto do miocárdio em pacientes com púrpura trombocitopênica idiopática

\begin{tabular}{|c|c|c|c|c|c|c|c|c|}
\hline Autor & $\begin{array}{c}\text { Idade } \\
(\text { anos }) / \operatorname{sexo}\end{array}$ & Plaquetas & Esteroide & Acesso & $\begin{array}{l}\text { Sangramento } \\
\text { maior }\end{array}$ & IE & $\begin{array}{l}\text { Antipla- } \\
\text { quetários }\end{array}$ & Heparina \\
\hline Fuchi $^{4}$ & $72 / \mathrm{F}$ & 59.000 & Sim & Femoral & Sim & Sim & Não & NF \\
\hline Stouffer ${ }^{5}$ & $77 / \mathrm{M}$ & 70.000 & Sim & Femoral & Sim & Não & $A+C$ & NF \\
\hline Yildiz $^{6}$ & $23 / F$ & 35.000 & Sim & Femoral & Não & Não & $A+C$ & NF \\
\hline $\mathrm{Kim}^{7}$ & $47 / F$ & 21.000 & Sim & Femoral & Não & Sim & $A+C$ & NF \\
\hline Kikuchi $^{8}$ & $68 / F$ & 22.000 & Não & Femoral & Não & Não & $\mathrm{T}$ & NF \\
\hline Gracia $^{9}$ & $37 / M$ & 39.000 & Não & Femoral & Não & Não & $A+C$ & NF \\
\hline Fong $^{10}$ & $71 / \mathrm{F}$ & 30.000 & Não & Radial & Não & Sim & C & Não \\
\hline
\end{tabular}

$\mathrm{A}$ = ácido acetilsalicílico; $\mathrm{C}=$ clopidogrel; $\mathrm{F}$ = feminino; $\mathrm{IE}=$ imunoglobulina endovenosa; $M$ = masculino; $N F=$ não-fracionada; $\mathrm{T}=$ ticlopidina.

percutânea $(4,7 \%$ vs. 3,4\%, OR 1,29, IC 95\% 0,87-1,94; $P=0,21) \cdot{ }^{12}$

\section{CONFLITO DE INTERESSES}

Os autores declararam inexistência de conflito de interesses relacionado a este manuscrito.

\section{REFERÊNCIAS}

1. Marques LGM, Furukawa MK, Leitão TP, Quiñones JLA, Queiroz FC, Tiossi RF, et al. Angioplastia transluminal coronariana em portador de púrpura trombocitopênica idiopática. Arq Bras Cardiol. 2005;84(4):337-9.

2. Segal JB, Powe NR. Prevalence of immune thrombocytopenia: analyses of administrative data. J Thromb Haemost. 2006; 4(11):2377-83.

3. Cines DB, Blanchette VS. Immune thrombocytopenic purpura. N Engl J Med. 2002;346(13):995-1008.

4. Fuchi T, Kondo T, Sase K, Takahashi M. Primary percutaneous transluminal coronary angioplasty performed for acute myocardial infarction in a patient with idiopathic thrombocytopenic purpura. Jpn Circ J. 1999;63(2):133-6.

5. Stouffer GA, Hirmerova J, Moll S, Rubery B, Napoli M, Ohman EM, et al. Percutaneous coronary intervention in a patient with immune thrombocytopenia purpura. Catheter Cardiovasc Interv. 2004;61(3):364-7.

6. Yildiz A, Coskun U, Batukan OE, Keskin K. Primary percutaneous coronary intervention for acute myocardial infarction in a young female with idiopathic thrombocytopenic purpura: a case report and review. Case Report Med. 2010;2010:854682. Epub 2010 Mar 24.

7. Kim JH, Park KU, Chun WJ, Kim SH, Nah DY. Primary percutaneous coronary intervention for acute myocardial infarction with idiopathic thrombocytopenic purpura: a case report. J Korean Med Sci. 2006;21(2):355-7.

8. Kikuchi S, Hayashi Y, Fujioka S, Kukita H, Ochi N. A case of intracoronary stent implanted for acute myocardial infarction in an elderly patient with idiopathic thrombocytopenic purpura. Nippon Ronen Igakkai Zasshi. 2002;39(1):88-93. [Article in Japanese]

9. Gracia MC, Cebollero IC, Lezcano JS, Osuna GG, Miguel JA, Peralta LP. Invasive treatment performed for acute myocardial infarction in a patient with immune thrombocytopenic purpura. Int J Cardiol. 2008;127(3):e183-5.

10. Fong MC, Chen KC, Leu HB, Chen LC. Coronary revascularization in a patient with immune thrombocytopenic purpura. J Chin Med Assoc. 2006;69(9):436-8.

11. European Association for Percutaneous Cardiovascular Interventions, Wijns W, Kolh P, Danchin N, Di Mario C, Falk $\mathrm{V}$, et al. Guidelines on myocardial revascularization: The Task Force on Myocardial Revascularization of the European Society of Cardiology (ESC) and the European Association for Cardio-Thoracic Surgery (EACTS). Eur Heart J. 2010;31(20): 2501-55.

12. Jolly SS, Amlani S, Hamon M, Yusuf S, Mehta SR. Radial versus femoral access for coronary angiography or intervention and the impact on major bleeding and ischemic events: a systematic review and meta-analysis of randomized trials. Am Heart J. 2009;157(1):132-40. 